\title{
Identification of chronic kidney disease patient characteristics influencing the renoprotective effects of febuxostat therapy: a retrospective follow-up study
}

Akinori Yamaguchi, Makoto Harada, Yosuke Yamada, Koji Hashimoto and Yuji Kamijo*

\begin{abstract}
Background: The ability of antihyperuricemic therapy to exert renoprotective effects in patients with chronic kidney disease (CKD) is controversial. In the present study, we studied patient characteristics that may mask favorable impact of antihyperuricemic therapy on the progression of CKD.

Methods: This was a single-center, retrospective, follow-up study. One-hundred and seventy-eight CKD patients with hyperuricemia who received febuxostat therapy were included in this study. Mean serum uric acid (mUA) level after treatment and changes in estimated glomerular filtration rate ( $\triangle \mathrm{eGFR}$ ) over 6 months were measured and their correlation was examined. Patients were divided into two groups based on $\mathrm{mUA}$, and their $\Delta \mathrm{eGFR}$ were compared. These analyses were evaluated in various subgroups.

Results: Febuxostat therapy markedly decreased UA level in any CKD stage patients without resulting in serious adverse events. eGFRs of CKD patients in the $\mathrm{mUA}<6.0 \mathrm{mg} / \mathrm{dl}$ group were maintained, whereas those in the $\mathrm{mUA} \geq 6.0 \mathrm{mg} / \mathrm{dl}$ group decreased. A significant inverse correlation was observed between mUA and $\triangle \mathrm{eGFR}$ $(r=-0.16, p=0.019)$. The renoprotective effects of febuxostat were significant in the following subgroups: male patients, age $<70$ years, systolic blood pressure $<130 \mathrm{mmHg}$, normal cholesterol levels, and absence of diabetes. Coexisting vascular risk factors appear to exert additive masking effects against febuxostat renoprotection.
\end{abstract}

Conclusions: The results of this study suggest that various vascular risk factors markedly attenuate the renoprotective effects of febuxostat.

Keywords: Chronic kidney disease, Febuxostat, Hyperuremia, Uric acid, Vascular risk factors

\section{Background}

Hyperuricemia has been reported to be a risk factor for kidney dysfunction [1-5]. The mechanism underlying the progression of kidney dysfunction by activation of xanthine oxidase $(\mathrm{XO})$ has been reported. Activation of $\mathrm{XO}$ increases reactive oxygen species (ROS) production and oxidative stress, as well as uric acid (UA) production, and causes vascular damage and organ dysfunction. Indeed, ROS is known to inactivate nitric oxide production and activates renin-angiotensin system, resulting in endothelial dysfunction and/or tubular injuries [6-8].

\footnotetext{
* Correspondence: yujibeat@shinshu-u.ac.jp

Department of Nephrology, Shinshu University School of Medicine, 3-1-1 Asahi, Matsumoto, Nagano 390-8621, Japan
}

Alternatively, UA salts activate inflammasomes, triggering inflammation and leading to the development of tubular injuries [9-11]. Antihyperuricemic XO inhibitors, including febuxostat and allopurinol, are believed to suppress renal dysfunction via oxidative stress reduction and suppression of endothelial dysfunction and tubular injuries. However, some studies reported that hyperuricemia is not significantly related to the progression of kidney dysfunction in patients with advanced Chronic Kidney Disease (CKD) [12, 13], and that a renoprotective effect via lowering of the serum UA level was not observed in CKD patients [14]. Thus, the renoprotective effect of XO inhibitors is controversial, and further investigation is required. 
We often find that CKD patients treated with antihyperuricemics show improved or maintained estimated glomerular filtration rate (eGFR) along with a reduction in serum UA level, whereas we also found cases showing none of the renoprotective effects. Since various risk factors for kidney dysfunction are involved in CKD progression, these factors could make it difficult to detect febuxostat-induced renoprotection.

In this study, we investigated whether antihyperuricemic therapy using febuxostat exerts renoprotective effects in CKD patients and which patient characteristics influence these renoprotective effects.

\section{Methods}

\section{Study design and patients}

This was a single-center, retrospective, observational study. Two-hundred thirty-two CKD patients underwent antihyperuricemic therapy using febuxostat at the Department of Nephrology in Shinshu University Hospital between May 2011 and October 2014. Patients were defined as having CKD if eGFR was $<60 \mathrm{ml} / \mathrm{min} / 1.73 \mathrm{~m}^{2}$ and/or urine protein was $>0.5 \mathrm{mg} /$ day, or both urinary blood and protein were present over 3 months. We excluded patients with acute kidney injuries (AKI), acute nephritis, or rapidly progressive glomerulonephritis from the current study. Fifteen patients discontinued the febuxostat treatment owing to adverse events during the follow-up period, and 19 patients required renal replacement therapy. Follow-up clinical data in 20 patients could not be obtained owing to change in hospital or discontinuation of attendance. A total of 178 cases could be analyzed. No patients died during the follow-up period. Among these 178 patients, 118 did not previously use any antihyperuricemic agents and 60 were switched from allopurinol or benzbromarone to febuxostat. In Japan, a low initial dosage (10 $\mathrm{mg} /$ day $)$ is recommended (maximum dose $60 \mathrm{mg} /$ day). Moreover, febuxostat is known to show an increased AUC in the patients with severe kidney dysfunction (GFR $<30 \mathrm{ml} / \mathrm{min}$ ) [15], and careful administration is necessary for these patients. For these reasons, febuxostat administration commenced at a dose of $10 \mathrm{mg} /$ day in the antihyperuricemicnaïve group, and 10 or $20 \mathrm{mg} /$ day in the group that was switched from other agents. The respective attending physicians determined dosage adjustments based on serum UA levels and its safety during the study period. All treatments patients received are considered standard care for their condition. Patient characteristics and clinical data were tabulated using medical record information. Changes in eGFR and serum UA levels at 6 months after febuxostat treatment initiation were analyzed statistically.

\section{Statistical analyses}

To examine the effect of febuxostat therapy on changes in serum UA levels and eGFR, those parameters were measured at the time of febuxostat prescription, as well as 3 months and 6 months after therapy initiation. The correlation between mean serum uric acid level after treatment (mUA) and the change of eGFR after 6 months $(6 \mathrm{~m} \Delta \mathrm{eGFR})$ was analyzed using Spearman's correlation. mUA was calculated as the mean serum UA level at 3 and 6 months. $6 \mathrm{~m} \triangle \mathrm{eGFR}$ was defined as the difference obtained by subtracting eGFR at treatment initiation from eGFR at 6 months. To investigate the effect of antihyperuricemic therapy for renal function lowering in CKD patients, we performed the correlation and ROC analyses using mUA and $6 \mathrm{~m} \triangle \mathrm{eGFR}$ data. Based on a calculated optimal cutoff level estimating progression of kidney dysfunction and standard goal of UA lowering therapy, the $\triangle \mathrm{eGFR}$ was analyzed by dividing patients into $\mathrm{mUA}<6.0 \mathrm{mg} / \mathrm{dl}$ and $\mathrm{mUA} \geq 6.0 \mathrm{mg} / \mathrm{dl}$ groups.

The correlation between mUA and $6 \mathrm{~m} \Delta$ eGFR was analyzed in all patients or in patient subgroups based on characteristics at the start of the therapy. Each patient's clinical data were collected from their medical records. The patient characteristics examined were sex, age ( $<70$ vs. $\geq 70$ years), systolic blood pressure $(<130$ vs. $\geq 130 \mathrm{mmHg}$ ), the presence or absence of an abnormal cholesterol level, the presence or absence of diabetes mellitus, eGFR (<30 vs. $\left.\geq 30 \mathrm{ml} / \mathrm{min} / 1.73 \mathrm{~m}^{2}\right)$, urine protein level ( $<0.5 \mathrm{vs.} \geq 0.5 \mathrm{~g} / \mathrm{gCre}$ ), and antihyperuricemic naive vs. agent switching group. The patients who were using a lipid-lowering drug or showed an abnormal level of LDL or HDL cholesterol (LDL cholesterol $\geq 140 \mathrm{mg} / \mathrm{dl}$, HDL cholesterol $<40 \mathrm{mg} / \mathrm{dl}$ ) were defined as having symptoms of abnormal cholesterol levels. The patients who were using an antidiabetic or presented above $6.2 \%$ of HbA1c (NGSP) in a blood examination were defined as diabetes mellitus.

The subgroups, in which a significant correlation between $\mathrm{mUA}$ and $6 \mathrm{~m} \triangle \mathrm{eGFR}$ was detected, were then divided into the $\mathrm{mUA}<6.0 \mathrm{mg} / \mathrm{dl}$ group and the $\mathrm{mUA} \geq 6.0 \mathrm{mg} / \mathrm{dl}$ group to compare $\triangle \mathrm{eGFR}$. We also analyzed the other subgroups, which were separated based on numbers of risk factors.

Mann-Whitney $U$ test was used to examine the statistical significance in continuous variables, and Fisher's exact test was used to examine category variables. Wilcoxon signed-rank test was used to examine the statistical significance in time course changes in continuous variables. SPSS Statistics v.22.0 J (IBM Corp., Armonk, NY, USA) analysis software was used in this study. Differences were considered statistically significant at $p<0.05$.

\section{Results}

Patient characteristics

Patient characteristics are presented in Table 1. Men accounted for $68 \%$ of the patient population, and the 
Table 1 Patient characteristics

\begin{tabular}{|c|c|c|c|c|}
\hline & All & $\mathrm{s} U \mathrm{~A}<6.0 \mathrm{mg} / \mathrm{dl}$ & $\mathrm{s} \cup \mathrm{A} \geq 6.0 \mathrm{mg} / \mathrm{dl}$ & $p$ value \\
\hline Patients, n (\%) & 178 & 78 & 100 & \\
\hline Male, n (\%) & $121(68 \%)$ & $42(54 \%)$ & 79 (79\%) & $<0.001$ \\
\hline Age, years (range) & $65(15-89)$ & $66(17-89)$ & $64(15-88)$ & n.s. \\
\hline Baseline systolic BP, mmHg (range) & $130(85-180)$ & $129(85-170)$ & $130(96-180)$ & n.s. \\
\hline Baseline diastolic BP, mmHg (range) & $76(45-110)$ & $74(50-110)$ & $76(45-106)$ & n.s. \\
\hline \multicolumn{5}{|l|}{ Primary diseases of CKD } \\
\hline Diabetic nephropathy, n (\%) & $33(19 \%)$ & $9(12 \%)$ & $24(24 \%)$ & n.s. \\
\hline Chronic nephritis, n (\%) & $36(20 \%)$ & $19(24 \%)$ & $17(17 \%)$ & n.s. \\
\hline Nephrosclerosis, n (\%) & $34(19 \%)$ & $14(18 \%)$ & $20(20 \%)$ & n.s. \\
\hline Others, n (\%) & $75(42 \%)$ & $36(46 \%)$ & 39 (39\%) & n.s. \\
\hline \multicolumn{5}{|l|}{ Complications } \\
\hline Diabetes mellitus, n (\%) & $45(25 \%)$ & $17(22 \%)$ & $28(28 \%)$ & n.s. \\
\hline Hypertension, n (\%) & 137 (77\%) & $56(72 \%)$ & $81(81 \%)$ & n.s. \\
\hline Abnormal cholesterol levels, n (\%) & $94(53 \%)$ & $34(44 \%)$ & $60(60 \%)$ & n.s. \\
\hline Coronary artery disease, n (\%) & $12(7 \%)$ & $4(5 \%)$ & $8(8 \%)$ & n.s. \\
\hline Estimated GFR at baseline, $\mathrm{ml} / \mathrm{min} / 1.73 \mathrm{~m}^{2}$ & $27.0(7.3-101.7)$ & $27.7(7.3-78.9)$ & $26.0(9-101.7)$ & n.s. \\
\hline Urine protein levels at baseline, g/gCre & $0.67(0.00-17.7)$ & $0.45(0.00-7.2)$ & $0.78(0.00-17.7)$ & n.s. \\
\hline Serum uric acid at baseline, mg/dl & $8.3(3.9-12.6)$ & $8.3(3.9-10.7)$ & $8.7(5.2-12.6)$ & 0.042 \\
\hline \multicolumn{5}{|l|}{ CKD stage } \\
\hline G1-2, n (\%) & $13(7 \%)$ & $6(8 \%)$ & $7(7 \%)$ & n.s. \\
\hline G3, n (\%) & $62(35 \%)$ & $29(37 \%)$ & $33(33 \%)$ & n.s. \\
\hline G4-5, n (\%) & $103(58 \%)$ & $43(55 \%)$ & $60(60 \%)$ & n.s. \\
\hline \multicolumn{5}{|l|}{ Antihyperuricemics before febuxostat } \\
\hline None, n (\%) & $118(66 \%)$ & $58(74 \%)$ & $60(60 \%)$ & n.s. \\
\hline Allopurinol, n (\%) & $44(25 \%)$ & $14(18 \%)$ & $30(30 \%)$ & n.s \\
\hline Benzbromarone, n (\%) & $14(8 \%)$ & $6(8 \%)$ & $8(8 \%)$ & n.s. \\
\hline Allopurinol and benzbromarone, n (\%) & $2(1 \%)$ & $0(0 \%)$ & $2(2 \%)$ & n.s. \\
\hline \multicolumn{5}{|l|}{ Febuxostat dose after 6 months } \\
\hline 10 mg/day, n (\%) & $103(58 \%)$ & $51(66 \%)$ & $52(52 \%)$ & n.s. \\
\hline 20 mg/day, n (\%) & $61(34 \%)$ & $22(28 \%)$ & $40(39 \%)$ & n.s. \\
\hline 40 mg/day, n (\%) & $14(8 \%)$ & $5(6 \%)$ & $9(9 \%)$ & n.s. \\
\hline
\end{tabular}

median age was 65 years. The median systolic and diastolic blood pressures at the start of febuxostat treatment were $130 \mathrm{mmHg}$ (range: $85-180$ ) and $76 \mathrm{mmHg}$ (range: 45-110), respectively. Primary diseases of CKD included diabetic nephropathy in 19\%, chronic nephritis in $20 \%$, and nephrosclerosis in $19 \%$ of patients. The proportion of complications of diabetes mellitus, hypertension, or abnormal cholesterol levels was 25,77 , and $53 \%$ of patients, respectively. A history of coronary artery disease was confirmed in $7 \%$ of patients. The median eGFR was $27 \mathrm{ml} / \mathrm{min} / 1.73 \mathrm{~m}^{2}$ (range: 7.3-101.7). Patient CKD stages at the start of treatment were $7 \%$ G1-2, 35\% G3, and 58\% G4-5. About 66\% of patients had not used any antihyperuricemics previously, with the remaining $34 \%$ using allopurinol or benzbromarone. Febuxostat dose after 6 months was $10 \mathrm{mg} /$ day in $58 \%, 20 \mathrm{mg} /$ day in $34 \%$, and $40 \mathrm{mg} /$ day in $8 \%$ of patients.

\section{Adverse events}

There were 15 cases wherein febuxostat treatment was discontinued during the follow-up period owing to adverse events $(6.5 \%$ of 232 cases, Additional file 1: Table S1). No serious adverse events developed in any of these cases, and discontinuation of the drug led to rapid disappearance of the adverse reaction in all cases. Compared to the group of patients who could continue the febuxostat treatment, the adverse event group included a significantly higher percentage of female 
patients (67\% of 15 cases). In most patients (93\% of 15 cases), the adverse events developed during the initial dosing of febuxostat at $10 \mathrm{mg} /$ day.

\section{Antihyperuricemic effect of febuxostat}

The changes in serum UA levels after febuxostat administration are shown in Fig. 1. The all patients, antihyperuricemic naive, and antihyperuricemic switched groups all showed significant decreases of serum UA levels at 3 and 6 months after treatment initiation. The mUA of all patients was $6.2 \pm 1.2 \mathrm{mg} / \mathrm{dl}$. Dividing the patients into subgroups based on their eGFR or urine protein level revealed that febuxostat exerted a significant antihyperuricemic effect, which were identical among the groups. Six a

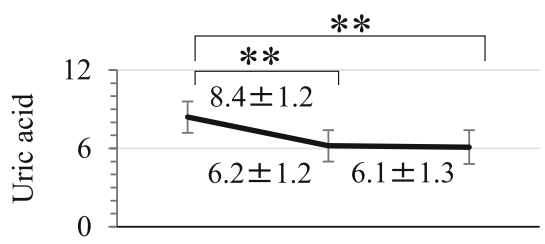

Rate of patients baseline 3 months 6 months with $\mathrm{UA}<6.0 \quad 4 \% \quad 43 \% \quad 47 \%$

b

Antihyperuricemic naïve

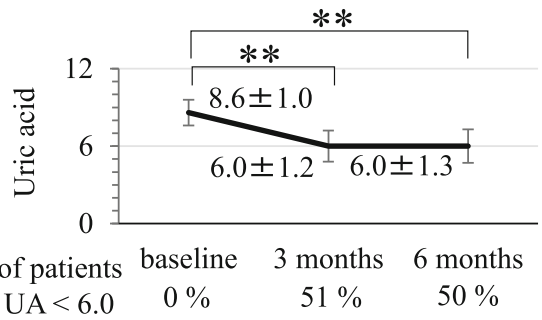

$\mathrm{C}$

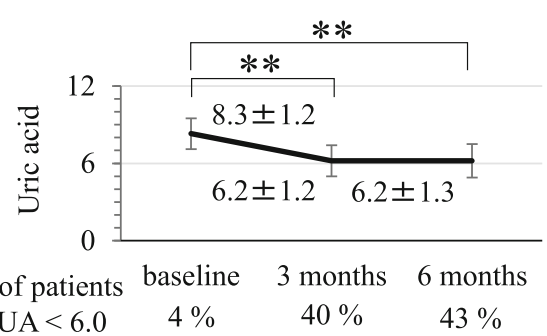

d

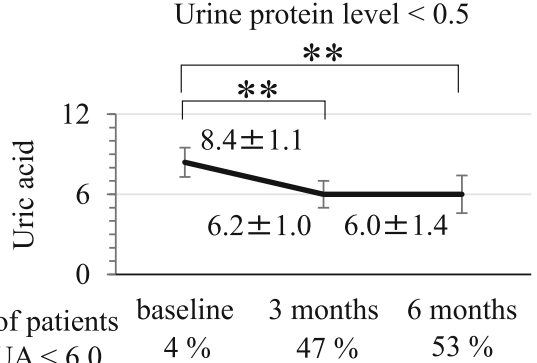

Antihyperuricemic switched

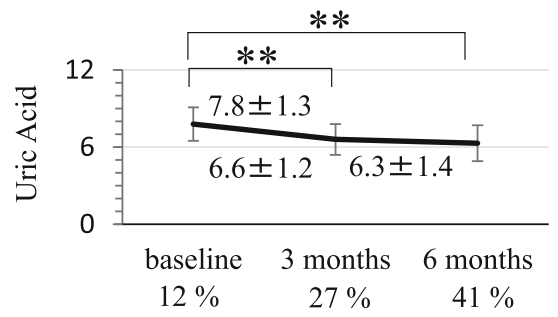

eGFR $\geq 30$

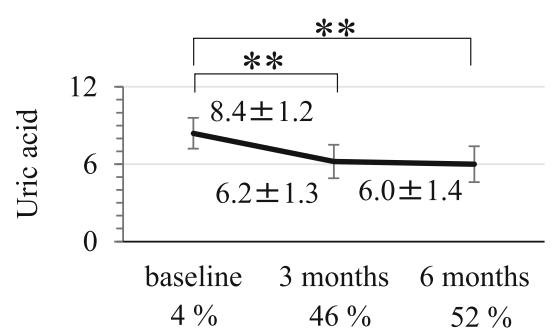

Urine protein level $\geq 0.5$

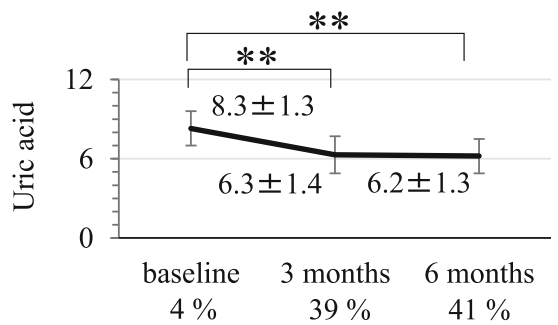

Fig. 1 Alteration of serum uric acid level due to febuxostat therapy. The changes in serum uric acid (UA) levels after febuxostat administration (baseline, 3 and 6 months after treatment initiation). a All patients $(n=178)$, $\mathbf{b}$ antihyperuricemic naïve $(n=118)$ vs. antihyperuricemic switched groups $(n=60)$, c eGFR $<30(n=75)$ vs. eGFR $\geq 30 \mathrm{ml} / \mathrm{min} / 1.73 \mathrm{~m}^{2}$ groups $(n=103)$, $\mathbf{d}$ urine protein level $<0.5(n=81)$ vs. urine protein level $\geq 0.5 \mathrm{~g} / \mathrm{gCre}$ groups $(n=92)$. The UA levels represent the mean \pm SD. ${ }^{* *} p<0.01$, compared with baseline UA level in each group (Wilcoxon signed-rank test) 
months after therapy, the serum UA level was lower than $6.0 \mathrm{mg} / \mathrm{dl}$ in $40-50 \%$ of patients in all groups.

\section{eGFR changes due to febuxostat therapy}

The scatter plot and receiver operating characteristic (ROC) curve using mUA and $6 \mathrm{~m} \triangle \mathrm{eGFR}$ data are shown in Additional file 2: Figure S1. A significant inverse correlation was detected between mUA and $6 \mathrm{~m} \triangle \mathrm{eGFR}$ in all patients, suggesting that the decrease of mUA leads to attenuation of CKD-induced eGFR decline. ROC analysis revealed that the calculated optimal cutoff level of mUA estimating progression of kidney dysfunction was $6.25 \mathrm{mg} / \mathrm{dl}$ (AUC 0.573, sensitivity 59.4\%, specificity $61.0 \%)$. Traditionally, the standard goal of antihyperuricemic therapy has been set at mUA $<6.0 \mathrm{mg} / \mathrm{dl}$ in most of the clinical studies, including a report assessing renoprotective effect of antihyperuricemics [16]. Since this traditional target UA level is very close to our calculated cutoff level (mUA $<6.25 \mathrm{mg}$ / $\mathrm{dl}$ ), mUA $<6.0 \mathrm{mg} / \mathrm{dl}$ (sensitivity $67.3 \%$, specificity $52.0 \%)$ was used as the cutoff level to be consistent with the past studies.

$\triangle$ eGFR measurements after febuxostat administration are shown in Fig. 2. No significant change was seen in $\triangle \mathrm{eGFR}$ at 3 and 6 months after treatment initiation when all patients were grouped together. However, dividing the patients into mUA $<6.0 \mathrm{mg} /$ $\mathrm{dl}$ and $\mathrm{mUA} \geq 6.0 \mathrm{mg} / \mathrm{dl}$ groups led to the detection of a significant eGFR decrease in the mUA $\geq 6.0 \mathrm{mg} / \mathrm{dl}$ group. No significant change in eGFR was noted in the mUA $<6.0 \mathrm{mg} / \mathrm{dl}$ group. Comparing the two groups, eGFR was significantly higher at 3 and 6 months in the mUA $<6.0 \mathrm{mg} / \mathrm{dl}$ group than in the $\mathrm{mUA} \geq 6.0 \mathrm{mg} / \mathrm{dl}$ group, indicating the renoprotective effects associated with lowering of serum UA levels.

All patients

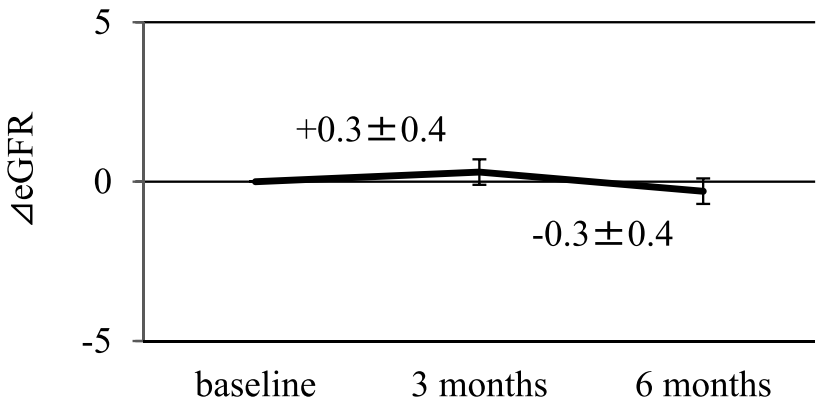

b The $\mathrm{mUA}<6.0 \mathrm{mg} / \mathrm{dl}$ group and the $\mathrm{mUA} \geq 6.0 \mathrm{mg} / \mathrm{dl}$ group

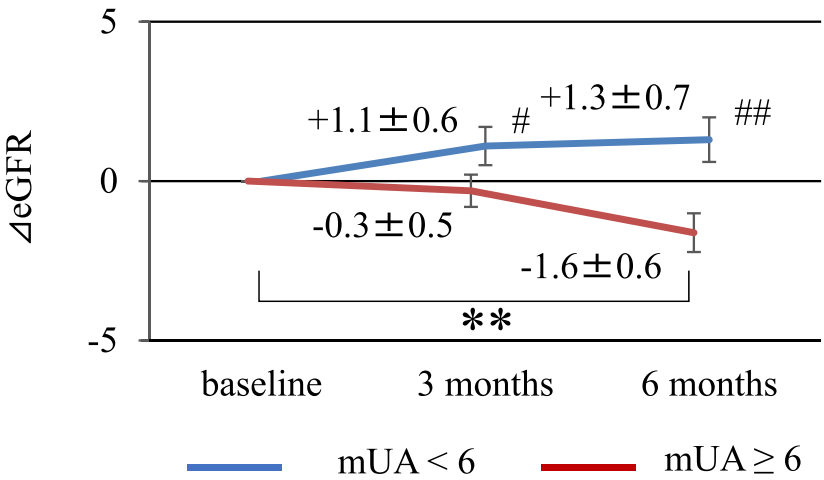

Fig. 2 Alteration of $\triangle \mathrm{eGFR}$ due to febuxostat therapy. The changes of eGFR ( $\triangle \mathrm{eGFR}$ ) after febuxostat administration (baseline, 3 and 6 months after treatment initiation). a All patients $(n=178)$. b The $\Delta$ eGFR was analyzed by dividing patients into mean serum uric acid level $(\mathrm{mUA})<6.0 \mathrm{mg} / \mathrm{dl}$ (blue line, $n=78$ ) vs. mUA $\geq 6.0 \mathrm{mg} / \mathrm{dl}$ (red line, $n=100$ ) groups. $\Delta$ eGFR represent the mean $\pm \mathrm{SE}$. $\Delta$ eGFR was defined as the difference obtained by subtracting baseline eGFR from post-treatment eGFR. ${ }^{* *} p<0.01$; compared with baseline UA level in each group (Wilcoxon signed-rank test). \# $p<0.05$, \#\# $p<0.01$; comparison between $\mathrm{mUA}<6.0$ and $\mathrm{mUA} \geq 6.0 \mathrm{mg} / \mathrm{dl}$ groups (Mann-Whitney $U$ test) 
Relationship between serum UA level and eGFR change in various subgroups

The relationship between mUA and $6 \mathrm{~m} \Delta \mathrm{eGFR}$ showed a wide variation when all patients were grouped together, resulting in a low correlation coefficient. Therefore, the correlation was examined relative to specific patient characteristics after dividing the patients into various subgroups (Additional file 3: Figure S2). A significant inverse correlation between mUA and $6 \mathrm{~m} \triangle \mathrm{eGFR}$ was detected in the following subgroups: male patients, patient age $<70$ years, systolic blood pressure $<130 \mathrm{mmHg}$, absence of abnormal cholesterol levels, and absence of diabetes mellitus. On the contrary, no inverse correlation was observed in the opposite subgroups: female patients, patient age $\geq 70$ years, systolic blood pressure $\geq 130 \mathrm{mmHg}$, abnormal cholesterol levels, and diabetes mellitus. These analyses indicate that effects of febuxostat therapy can be affected by various vascular risk factors. Additionally, no significant inverse correlation between mUA and $6 \mathrm{~m} \triangle \mathrm{eGFR}$ was detected in subgroups divided based on eGFR $30 \mathrm{ml} / \mathrm{min} / 1.73 \mathrm{~m}^{2}$, urine protein levels $0.5 \mathrm{~g} /$ gCre, or antihyperuricemic naïve and agent switching groups (data not shown).

The subgroups showing an inverse correlation between mUA and $6 \mathrm{~m} \triangle \mathrm{e} G F R$ were further divided based on the mUA level to compare their $6 \mathrm{~m} \Delta \mathrm{eGFR}$ (Figure 3 ). In the subgroups of men, aged $<70$ years, systolic blood pressure $<130 \mathrm{mmHg}$, normal cholesterol levels, and absence of diabetes, a significant increase of eGFR was detected in the mUA $<6.0 \mathrm{mg} / \mathrm{dl}$ group compared to that in the $\mathrm{mUA} \geq 6.0 \mathrm{mg} / \mathrm{dl}$ group. In these subgroups, a significant difference in $6 \mathrm{~m} \triangle \mathrm{eGFR}$ was detected between the $\mathrm{mUA}<6.0$ and $\mathrm{mUA} \geq 6.0 \mathrm{mg} / \mathrm{dl}$ groups. On the contrary, the opposite subgroups including women, aged $\geq 70$ years, systolic blood pressure $\geq 130 \mathrm{mmHg}$, abnormal cholesterol levels, and diabetes, exhibited slight changes in eGFR. In these subgroups with vascular risk factors, a significant difference in $6 \mathrm{~m} \Delta \mathrm{eGFR}$ was obscure between $\mathrm{mUA}<6.0$ and $\mathrm{mUA} \geq 6.0 \mathrm{mg} / \mathrm{dl}$ groups.

\section{Relationship between mUA level and eGFR change in subgroups divided based on the number of vascular risk factors}

The correlations between mUA and $6 \mathrm{~m} \Delta \mathrm{eGFR}$ in subgroups divided based on the number of these vascular risk factors are shown in Additional file 4: Figure S3. In the subgroup without vascular risk factors, there is a strong significant inverse correlation between mUA and $6 \mathrm{~m} \Delta$ eGFR $(r=-0.4, p=0.029, n=30)$. In the subgroups with one to two vascular risk factors, a significant inverse correlation between mUA and $6 \mathrm{~m} \triangle \mathrm{eGFR}$ became obscure, and the correlation completely vanished in the subgroup with more than three cardiovascular risk factors.
The changes of eGFR in each subgroup, which were further divided based on mUA levels, are shown in Fig. 4. In the subgroups with one or less risk factors, eGFR increased in mUA $<6.0$ groups, and significant differences in $6 \mathrm{~m} \triangle \mathrm{eGFR}$ were detected between mUA $<6.0$ group and $\mathrm{mUA} \geq 6.0$ group. In the subgroups with more two risk factors, improvement of eGFR via hyperuricemic therapy, as well as significant differences in $6 \mathrm{~m} \triangle \mathrm{e} G F R$ between $\mathrm{mUA}<6.0$ group and $\mathrm{mUA} \geq 6.0$ group, were not detected.

\section{Discussion}

Attempts to control serum UA levels using previous antihyperuricemic medicines, including benzbromarone and allopurinol, in patients with kidney dysfunction, have proved difficult. Since benzbromarone is an agent that enhances urinary excretion of UA, its antihyperuricemic effect is attenuated by kidney dysfunction. Another $\mathrm{XO}$ inhibitor, allopurinol, is mainly excreted by urine; therefore, its serum concentration and adverse events are increased in the patients with kidney dysfunction, requiring dose reduction from an early phase of CKD and resulting in poor control of serum UA levels. Since febuxostat is excreted via dual excretion pathways, urine $49.1 \%$ and fecal $44.9 \%$ [17], febuxostat can exert strong antihyperuricemic effect without serious adverse events even for patients with kidney dysfunction without changing dosage $[18,19]$. Although careful febuxostat administration is necessary for patients with severe kidney dysfunction (GFR $<30 \mathrm{ml} / \mathrm{min}$ ), its metabolic characteristics are advantageous compared to those of allopurinol. Indeed, this study demonstrates that febuxostat therapy is effective in controlling serum UA levels in patients at any CKD stage. The percentage of patients for whom febuxostat could be administered continuously for 6 months was high, as the incidence of serious adverse events was very low. In the very small number of CKD patients who developed an adverse event, the symptoms improved rapidly after drug discontinuation. These results are consistent with reports stating that febuxostat treatment exerts a more potent antihyperuricemic effect with an equivalent incidence of adverse events compared to allopurinol in patients with kidney dysfunction $[18,19]$. Therefore, these results indicate that febuxostat treatment is useful for lowering serum UA levels in CKD patients.

Many studies have reported that hyperuricemia is a risk factor for the progression of kidney dysfunction in the general population, diabetes mellitus, AKI and CKD patients $[1-5]$. Therefore, antihyperuricemic therapy is expected to contribute to kidney protection. In this study, we showed that the decline of eGFR was remarkable in CKD patients with poor control of UA level $(\mathrm{mUA} \geq 6.0 \mathrm{mg} / \mathrm{dl})$, whereas it was likely to be 
$\mathrm{a}$

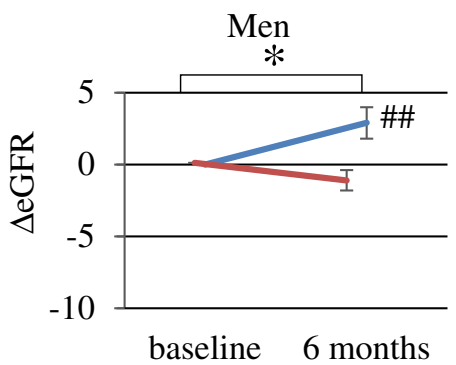

b

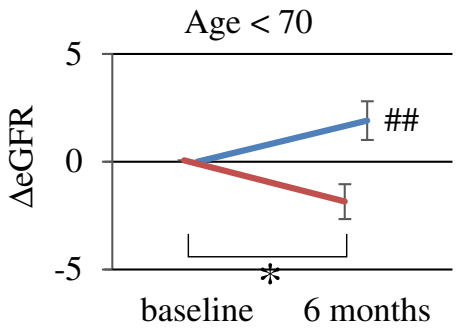

c

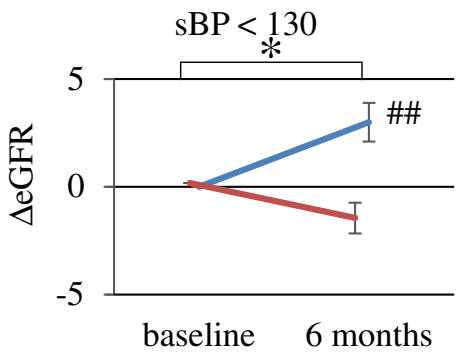

d

Without abnormal cholesterol levels

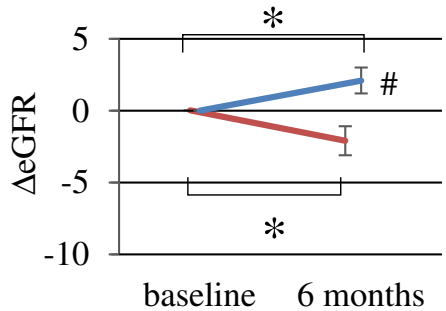

e

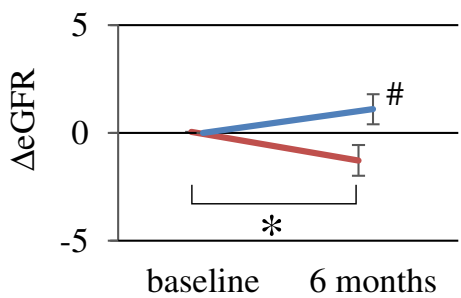

Women
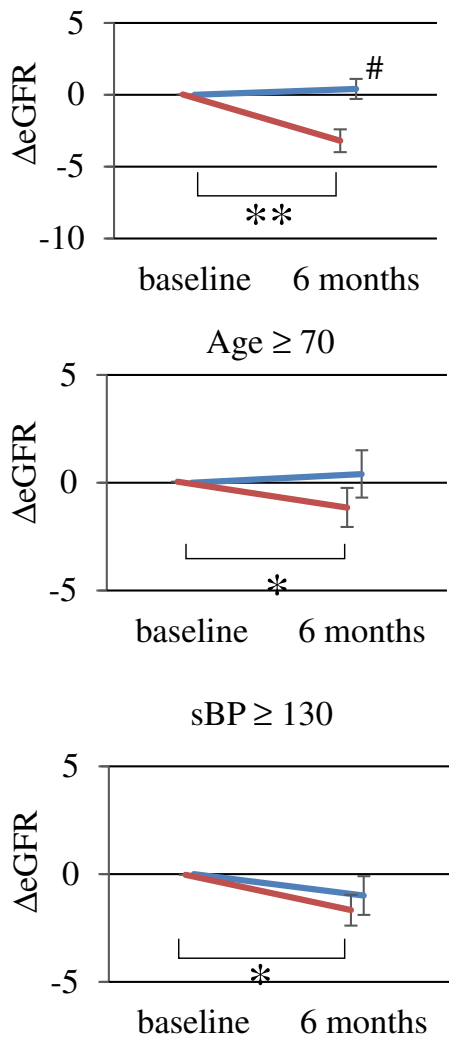

With abnormal cholesterol levels

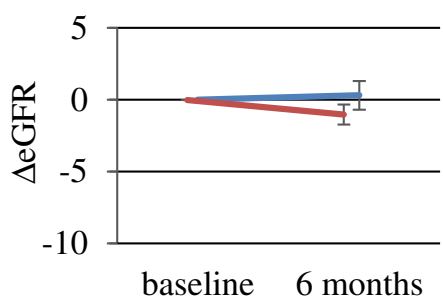

With diabetes mellitus

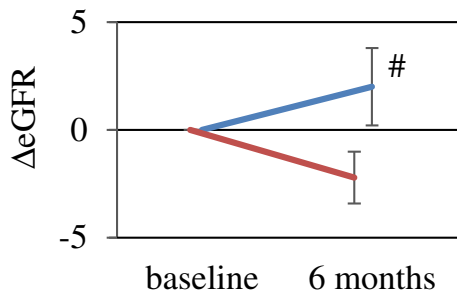

Fig. 3 (See legend on next page.) 
(See figure on previous page.)

Fig. 3 Difference in $6 \mathrm{~m} \Delta$ eGFR in mUA $<6.0$ vs. $\geq 6.0$ groups in various subgroups. $\Delta$ eGFR after 6 months ( $6 \mathrm{~m} \Delta$ eGFR) were analyzed by dividing patients into mean serum uric acid (mUA) level $<6.0 \mathrm{mg} / \mathrm{dl}$ vs. $\mathrm{mUA} \geq 6.0 \mathrm{mg} / \mathrm{dl}$ groups. The analyses were performed in various subgroups. a Men $(n=121)$ vs. Women $(n=57)$, b Age $<70(n=107)$ vs. age $\geq 70$ years $(n=71)$, c systolic blood pressure $(s B P)<130(n=95) v s . s B P \geq 130 \mathrm{mmHg}$ ( $n=83)$, d normal cholesterol levels $(n=84)$ vs. abnormal cholesterol levels $(n=94)$, e absence of diabetes mellitus $(n=133)$ vs. diabetes mellitus $(n=45) .6 \mathrm{~m} \Delta$ eGFR represent the mean \pm SE. ${ }^{*} p<0.05,{ }^{* *} p<0.01$, compared with baseline UA level in each group (Wilcoxon signed-rank test). $\# p<0.05$, \#\# $p<0.01$; comparison between $\mathrm{mUA}<6.0$ and $\mathrm{mUA} \geq 6.0 \mathrm{mg} / \mathrm{dl}$ groups (Mann-Whitney $\mathrm{U}$ test)

ameliorated by febuxostat therapy in patients with good control of UA level $(\mathrm{mUA}<6.0 \mathrm{mg} / \mathrm{dl})$. In addition, we detected an inverse correlation between mUA and $6 \mathrm{~m} \triangle \mathrm{eGFR}$, suggesting that further decreases in mUA after febuxostat administration contribute to suppression of CKD-induced eGFR decline. These findings support the hypothesis that lowering serum UA level is renoprotective. These results are consistent with previous studies reporting that antihyperuricemic therapy in patients with CKD ameliorates eGFR reduction [20, 21]. However, the renoprotective effects of antihyperuricemic therapy in CKD patients are controversial in several

a No vascular risk factors $(\mathrm{n}=30)$

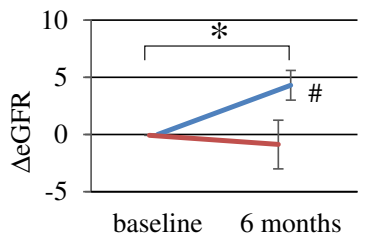

b

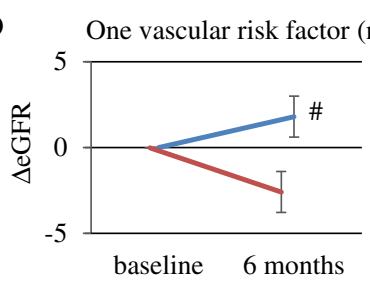

\begin{tabular}{lc} 
Vascular risk factors & $\mathrm{n}$ \\
\hline $\mathrm{Age} \geq 70$ & 16 \\
$\mathrm{sBP} \geq 130$ & 17 \\
Abnormal cholesterol levels & 20 \\
Diabetes mellitus & 2
\end{tabular}

C Two vascular risk factors $(n=48)$

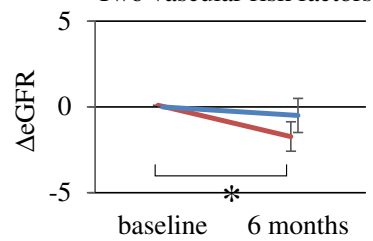

\begin{tabular}{lc} 
Vascular risk factors & $\mathrm{n}$ \\
\hline Age $\geq 70$ & 24 \\
$\mathrm{sBP} \geq 130$ & 27 \\
Abnormal cholesterol levels & 33 \\
Diabetes mellitus & 12
\end{tabular}

d Three or four vascular risk factors $(n=45)$

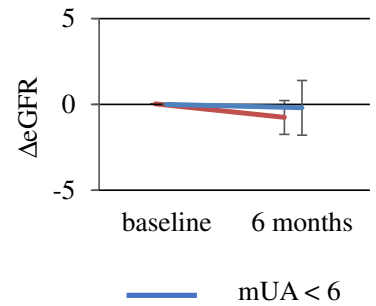

\begin{tabular}{ll} 
Vascular risk factors & $\mathrm{n}$ \\
\hline Age $\geq 70$ & 31 \\
$\mathrm{sBP} \geq 130$ & 39 \\
Abnormal cholesterol levels & 41 \\
Diabetes mellitus & 31
\end{tabular}

$m U A \geq 6$

Fig. 4 Difference in $6 \mathrm{~m} \Delta$ eGFR in subgroups divided based on the number of vascular risk factors. $\Delta$ eGFR after 6 months ( $6 \mathrm{~m} \Delta \mathrm{eGFR}$ ) were analyzed by dividing patients into mean serum uric acid (mUA) level $<6.0 \mathrm{mg} / \mathrm{dl} \mathrm{vs.} \mathrm{mUA} \geq 6.0 \mathrm{mg} / \mathrm{dl}$ groups. The analyses were performed in subgroups divided based on the number of vascular risk factors. a No vascular risk factors $(n=30)$, b with one vascular risk factors ( $n=55)$, c with two vascular risk factors $(n=48)$, $\mathbf{d}$ with three or four vascular risk factors $(n=45)$. $\Delta$ eGFR represent the mean \pm SE. ${ }^{*} p<0.05$, compared with baseline UA level in each group (Wilcoxon signed-rank test). \# $p<0.05$; comparison between $\mathrm{mUA}<6.0$ and $\mathrm{mUA} \geq 6.0 \mathrm{mg} / \mathrm{dl} \mathrm{groups}$ (Mann-Whitney U test) 
small-scale randomized controlled trials (RCTs) and various follow-up studies involving CKD patients. Two placebo-controlled small-scale RCTs in which allopurinol or febuxostat were used reported that CKD-induced eGFR decline was suppressed more in the antihyperuricemic group than in the placebo group [20, 21]. Levy DG et al. performed antihyperuricemic therapy for 3 years using allopurinol, febuxostat, or probenecid for patients with hyperuricemia, and found that renal dysfunction could be suppressed in patients with controlled UA levels at $<6 \mathrm{mg} / \mathrm{dL}$ [16]. In our study, we also found that deterioration of kidney function could be suppressed in patients with UA levels controlled at lower than $6 \mathrm{mg} / \mathrm{dl}$, supporting the above reports. In contrast, a RCT involving 40 patients with IgA nephropathy and CKD stage 1-3 reported no renoprotective effects of a 6-month allopurinol therapy course [14]. The lack of consensus among many clinical studies regarding a renoprotective effect by antihyperuricemic therapy may be due to the differences of patient characteristics in each study.

The current subgroup analyses demonstrated that patients with parameters including male, $<70$ years, systolic blood pressure $<130 \mathrm{mmHg}$, no abnormal cholesterol levels, or no diabetes mellitus are more likely to show an inverse correlation between mUA level and $6 \mathrm{~m} \triangle \mathrm{eGFR}$, as well as amelioration of eGFR decline by UA lowering, than their opposite subgroups. This result suggests that the renoprotective effects of febuxostat differ among CKD patients. The factors masking the renoprotective effects detected in this study, including aging, hypertension, hyperlipidemia, and diabetes mellitus, are important vascular risk factors causing endothelial dysfunction and arteriosclerosis. $\mathrm{XO}$ activation and hyperuricemia are also thought to be vascular injury factors. $\mathrm{XO}$ activation increases oxidative stress, suppresses nitric oxide production, and activates the renin-angiotensin system [6-8]. Hyperuricemia is reported to induce arteriolopathy of preglomerular vessels, which impairs endothelial function and the autoregulatory response of afferent arterioles, resulting in glomerular hypertension [8]. Antihyperuricemic therapy using febuxostat is expected to improve these vascular events induced by $\mathrm{XO}$ activation and hyperuricemia; however, its vascular protective effects may be masked by other vascular risk factors. The current results demonstrate that eGFR is likely to decrease despite optimal control of the UA level in CKD patients with various vascular risk factors, and that coexisting vascular risk factors exert additive masking effects against febuxostat renoprotection. To detect the reliable renoprotective effects of antihyperuricemic therapy, clinical studies in which control and interventional groups have small number of background vascular risk factors will be needed in the future.
The current findings do not negate the therapeutic significance of febuxostat treatment for CKD patients with various risk factors such as diabetes mellitus. Zoppini et al. reported that hyperuricemia is an independent risk factor for CKD progression in patients with type 2 diabetes [2]. We believe that antihyperuricemic therapy is an important interventional strategy to protect kidney function even in patients with diabetes mellitus, because it is expected to ameliorate additional vascular damage induced by hyperuricemia.

Limitations of this study include a small number of subjects from a short duration, single-center retrospective follow-up study. The analyses with small $n$-number and short-duration follow-up might show borderline significance values, especially in subgroup analyses. However, we believe that the findings of the current study would provide useful information for clinical studies in the future, by identifying the patient characteristics influencing the renoprotective effects of febuxostat therapy. Further clarification of the differences in the renoprotection effects by antihyperuricemic therapy among various patient subgroups will require future examination in large-scale, extendedduration, multi-center prospective studies. Second, the dosage of febuxostat in Japan differs from that in other countries due to safety assessment and the dose ranging studies. The proportions of patients with serum $\mathrm{UA}<6.0 \mathrm{mg} / \mathrm{dl}$ were $40 \mathrm{mg}, 56 \% ; 80 \mathrm{mg}, 76 \%$; and $120 \mathrm{mg}, 94 \%$ in the dose ranging study in USA [22], whereas they were $20 \mathrm{mg}, 46.5 \%$; $40 \mathrm{mg}, 82.9 \% ; 60 \mathrm{mg}$, 83.3\%; and $80 \mathrm{mg}, 87.8 \%$ in Japan [23]. These findings suggest that low-dose febuxostat therapy can lower serum UA levels in Japanese patients to a greater extent than in European and Americans patients. From the results of the APEX, FACT, and CONFIRMS studies $[17,18,24]$, febuxostat dose was set at $40-80 \mathrm{mg} /$ day in USA and $80-120 \mathrm{mg} /$ day in Europe, whereas the initial dose was set at $10 \mathrm{mg} /$ day, with careful gradual dose increase of febuxostat (maximum $60 \mathrm{mg} /$ day), in Japan based on the results of the allopurinol-controlled comparative studies and a long-term study [25-27]. This suggested a possible difference in antihyperuricemic effect due to factors such as genetic differences, body mass index, or dietary habits. Therefore, the result of the present study was not directly applicable to patients of other countries. Third, the current study included only a small of number female patients, in whom a high number of adverse events were reported, making the evaluation of the clinical effects of febuxostat therapy in female patients inconclusive. Therefore further research for female patients is necessary. In addition, factors not examined in this study might have influenced the study results; therefore, careful interpretation of the results would be required. 


\section{Conclusions}

Febuxostat therapy was effective in controlling the UA level in patients at any CKD stage and was highly safe. In the well-controlled UA level (mUA $<6.0 \mathrm{mg} / \mathrm{dl}$ ) group, antihyperuricemic therapy using febuxostat may exert renoprotective effects. The effects are more likely to be detected in patients free of vascular risk factors, such as aging, hypertension, hyperlipidemia, and diabetes mellitus.

\section{Additional files}

Additional file 1: Table S1. Adverse events. There were 15 cases wherein febuxostat treatment was discontinued owing to various adverse events. In most patients, the adverse events developed during the initial dosing of febuxostat at $10 \mathrm{mg} /$ day. (DOCX $18 \mathrm{~kb}$ )

Additional file 2: Figure S1. Correlation between mUA and $6 \mathrm{~m} \Delta \mathrm{eGFR}$, and ROC curve indicating mUA cutoff level estimating CKD progression. (a) Scatter plot indicating the correlation between mean serum uric acid (mUA) level and $\triangle$ eGFR after 6 months ( $6 m \Delta$ eGFR) $(n=178)$. A correlation coefficient $(r)$ and $p$-value $(\mathrm{p})$ were analyzed using Spearman's correlation analysis. (b) ROC analysis revealed that the calculated optimal cutoff level of mUA estimating progression of kidney dysfunction was $6.25 \mathrm{mg} / \mathrm{dl}$ (AUC 0.573, sensitivity 59.4\%, specificity 61.0\%). (PPTX 83 kb)

Additional file 3: Figure S2. Correlation between $\mathrm{mUA}$ and $6 \mathrm{~m} \Delta \mathrm{eGFR}$ in various subgroups. Scatter plots indicating the correlation between mean serum uric acid (mUA) level and $\triangle$ eGFR after 6 months $(6 \mathrm{~m} \Delta \mathrm{eGFR})$ in various subgroups. (a) Men $(n=121)$ vs. Women $(n=57)$, (b) Age $<70$ ( $n=107)$ vs. age $\geq 70$ years $(n=71)$, (c) systolic blood pressure $(\mathrm{sBP})<130(n=95)$ vs. $\mathrm{sBP} \geq 130 \mathrm{mmHg}(n=83)$, (d) normal cholesterol levels ( $n=84$ ) vs. abnormal cholesterol levels $(n=94)$, (e) absence of diabetes mellitus ( $n=133)$ vs. diabetes mellitus $(n=45)$. A correlation coefficient $(r)$ and

p-value (p) were analyzed using Spearman's correlation analysis. (PPTX $151 \mathrm{~kb}$ )

Additional file 4: Figure S3. Correlation between $\mathrm{mUA}$ and $6 \mathrm{~m} \Delta \mathrm{eGFR}$ in subgroups divided based on the number of vascular risk factors. Scatter plots indicating the correlation between mean serum uric acid ( $m \cup A$ ) level and $\triangle$ eGFR after 6 months ( $6 \mathrm{~m} \Delta \mathrm{eGFR}$ ) in subgroups divided based on the number of vascular risk factors. (a) No vascular risk factors $(n=30)$, (b) one vascular risk factor $(n=55)$, (c) two vascular risk factors $(n=48)$, (d) three or four vascular risk factors $(n=45)$. A correlation coefficient $(r)$ and $p$-value $(p)$ were analyzed using Spearman's correlation analysis. (PPTX 99 kb)

\section{Abbreviations}

$6 \mathrm{~m} \triangle \mathrm{eGFR}$ : The change in eGFR after 6 months; AKI: Acute kidney injury; CKD: Chronic kidney diseases; eGFR: Estimated glomerular filtration rate; mUA: Mean uric acid; RCT: Randomized controlled trials; ROC: Receiver operating characteristic; ROS: Reactive oxygen species; UA: Uric acids; XO: Xanthine oxidase; $\triangle \mathrm{eGFR}$ : The change in eGFR

\section{Acknowledgements}

We would like to thank Editage (www.editage.jp) for English language editing.

\section{Funding}

This study did not receive any funding

\section{Availability of data and materials}

The datasets used and analyzed during the current study are available from the corresponding author on reasonable request.

\section{Authors' contributions}

$\mathrm{AY}$ and $\mathrm{MH}$ proposed the concept of the study. AY collected the data. AY, $M H, Y Y$, and $K H$ analyzed and interpreted the data. AY and YK wrote the manuscript. All authors read and approved the final manuscript.

\section{Competing interests}

The authors declare that they have no competing interests.

\section{Consent for publication}

Not applicable.

\section{Ethics approval and consent to participate}

In this study, all treatments patients received are considered standard care for their condition. The study protocol complied with the guidelines of the 2013 version of the Declaration of Helsinki, and was approved by the Committee for Medical Ethics of Shinshu University School of Medicine (approval number: 3219). This ethics committee of Shinshu University waived the need for written informed consent because of the retrospective design; however, the committee requested that the research subjects be notified of the purpose of utilization of clinical information and allowed to refuse participation via a comprehensive explanatory form. The handouts of this comprehensive explanatory form were prepared for examination room reception, and waiting room and could be obtained freely; the posters of this form were put up on bulletin boards in the Shinshu University Hospital. Patients could receive additional explanation of the study protocol, and they were allowed to refuse study participation at any point. This opt-out policy is based on the Japanese Ethical Guidelines for Medical and Health Research Involving Human Subjects.

\section{Publisher's Note}

Springer Nature remains neutral with regard to jurisdictional claims in published maps and institutional affiliations.

Received: 29 December 2016 Accepted: 3 May 2017

Published online: 18 May 2017

\section{References}

1. Iseki K, Ikemiya Y, Inoue T, Iseki C, Kinjo K, Takishita S. Significance of hyperuricemia as a risk factor for developing ESRD in a screened cohort. Am J Kidney Dis. 2004:44:642-50.

2. Zoppini G, Targher G, Chonchol M, Ortalda V, Abaterusso C, Pichiri I, et al. Serum uric acid levels and incident chronic kidney disease in patients with type 2 diabetes and preserved kidney function. Diabetes Care. 2012;35:99-104.

3. Koratala A, Singhania G, Alquadan KF, Shimada M, Johnson RJ, Ejaz AA. Serum uric acid exhibits inverse relationship with estimated glomerular filtration rate. Nephron. 2016;134:231-7.

4. Ohno I, Hosoya T, Gomi H, Ichida K, Okabe H, Hikita M. Serum uric acid and renal prognosis in patients with IgA nephropathy. Nephron. 2001;87:333-9.

5. Syrjänen J, Mustonen J, Pasternack A. Hypertriglyceridaemia and hyperuricaemia are risk factors for progression of $\lg A$ nephropathy. Nephrol Dial Transplant. 2000;15:34-42.

6. Yu MA, Sánchez-Lozada LG, Johnson RJ, Kang DH. Oxidative stress with an activation of the renin-angiotensin system in human vascular endothelial cells as a novel mechanism of uric acid-induced endothelial dysfunction. J Hypertens. 2010;28:1234-42.

7. Tsuda H, Kawada N, Kaimori JY, Kitamura H, Moriyama T, Rakugi H, et al. Febuxostat suppressed renal ischemia-reperfusion injury via reduced oxidative stress. Biochem Biophys Res Commun. 2012;427:266-72.

8. Sánchez-Lozada LG, Tapia E, Santamaría J, Avila-Casado C, Soto V, Nepomuceno T, et al. Mild hyperuricemia induces vasoconstriction and maintains glomerular hypertension in normal and remnant kidney rats. Kidney Int. 2005;67:237-47.

9. Maejima I, Takahashi A, Omori H, Kimura T, Takabatake Y, Saitoh T, et al. Autophagy sequesters damaged lysosomes to control lysosomal biogenesis and kidney injury. EMBO J. 2013;32:2336-47.

10. Misawa T, Takahama M, Kozaki T, Lee H, Zou J, Saitoh T, et al. Microtubuledriven spatial arrangement of mitochondria promotes activation of the NLRP3 inflammasome. Nat Immunol. 2013:14:454-60.

11. Anders HJ, Muruve DA. The inflammasomes in kidney disease. J Am Soc Nephrol. 2011:22:1007-18.

12. Madero M, Sarnak MJ, Wang X, Greene T, Beck GJ, Kusek JW, et al. Uric acid and long-term outcomes in CKD. Am J Kidney Dis. 2009;53:796-803.

13. Liu WC, Hung CC, Chen SC, Yeh SM, Lin MY, Chiu YW, et al. Association of hyperuricemia with renal outcomes, cardiovascular disease, and mortality. Clin J Am Soc Nephrol. 2012;7:541-8.

14. Shi Y, Chen W, Jalal D, Li Z, Chen W, Mao H, et al. Clinical outcome of hyperuricemia in IgA nephropathy: a retrospective cohort study and randomized controlled trial. Kidney Blood Press Res. 2012;35:153-60. 
15. Mayer MD, Khosravan R, Vernillet L, Wu JT, Joseph-Ridge N, Mulford DJ. Pharmacokinetics and pharmacodynamics of febuxostat, a new non-purine selective inhibitor of xanthine oxidase in subjects with renal impairment. Am J Ther. 2005;12:22-34.

16. Levy GD, Rashid N, Niu F, Cheetham TC. Effect of urate-lowering therapies on renal disease progression in patients with hyperuricemia. J Rheumatol. 2014;41:955-62.

17. Grabowski BA, Khosravan R, Vernillet L, Mulford DJ. Metabolism and excretion of [14C] febuxostat, a novel nonpurine selective inhibitor of xanthine oxidase, in healthy male subjects. J Clin Pharmacol. 2011;51:189-201.

18. Schumacher HR Jr, Becker MA, Wortmann RL, Macdonald PA, Hunt B, Streit et al. Effects of febuxostat versus allopurinol and placebo in reducing serum urate in subjects with hyperuricemia and gout: a 28-week, phase III, randomized, double-blind, Parallel-Group Trial. Arthritis Rheum. 2008;59:1540-8.

19. Becker MA, Schumacher HR, Espinoza LR, Wells AF, MacDonald P, Lloyd E, et al. The urate-lowering efficacy and safety of febuxostat in the treatment of the hyperuricemia of gout: the CONFIRMS trial. Arthritis Res Ther. 2010;12:R63

20. Goicoechea M, de Vinuesa SG, Verdalles U, Ruiz-Caro C, Ampuero J, Rincón A, et al. Effect of allopurinol in chronic kidney disease progression and cardiovascular risk. Clin J Am Soc Nephrol. 2010;5:1388-93.

21. Sircar D, Chatterjee S, Waikhom R, Golay V, Raychaudhury A, Chatterjee S, et al. Efficacy of febuxostat for slowing the GFR decline in patients with CKD and asymptomatic hyperuricemia: a 6-month, double-blind, randomized, Placebo-Controlled Trial. Am J Kidney Dis. 2015;66:945-50.

22. Becker MA, Schumacher HR Jr, Wortmann RL, MacDonald PA, Palo WA, Eustace $D$, et al. Febuxostat, a novel nonpurine selective inhibitor of xanthine oxidase: a twenty-eight-day, multicenter, phase II, randomized, double-blind, placebo-controlled, dose-response clinical trial examining safety and efficacy in patients with gout. Arthritis Rheum. 2005;52:916-23.

23. Kamatani N, Fujimori S, Hada T, Hosoya T, Kohri K, Nakamura T, et al. Placebo-controlled double-blind dose-response study of the non-purineselective xanthine oxidase inhibitor febuxostat (TMX-67) in patients with hyperuricemia (including gout patients) in japan: late phase 2 clinical study. J Clin Rheumatol. 2011;17:S35-43.

24. Becker MA, Schumacher HR Jr, Wortmann RL, MacDonald PA, Eustace D, Palo WA, et al. Febuxostat compared with allopurinol in patients with hyperuricemia and gout. N Engl J Med. 2005;353:2450-61.

25. Kamatani N, Fujimori S, Hada T, Hosoya T, Kohri K, Nakamura T, et al. An allopurinol-controlled, multicenter, randomized, open-label, parallel betweengroup, comparative study of febuxostat (TMX-67), a non-purine-selective inhibitor of xanthine oxidase, in patients with hyperuricemia including those with gout in Japan: phase 2 exploratory clinical study. J Clin Rheumatol. 2011;17:S44-9.

26. Kamatani N, Fujimori S, Hada T, Hosoya T, Kohri K, Nakamura T, et al. An allopurinol-controlled, randomized, double-dummy, double-blind, parallel between-group, comparative study of febuxostat (TMX-67), a non-purineselective inhibitor of xanthine oxidase, in patients with hyperuricemia including those with gout in Japan: phase 3 clinical study. J Clin Rheumatol. 2011;17:S13-8.

27. Kamatani N, Fujimori S, Hada T, Hosoya T, Kohri K, Nakamura T, et al. Multicenter, open-label study of long-term administration of febuxostat (TMX-67) in Japanese patients with hyperuricemia including gout. J Clin Rheumatol. 2011;17:S50-6.

\section{Submit your next manuscript to BioMed Central and we will help you at every step:}

- We accept pre-submission inquiries

- Our selector tool helps you to find the most relevant journal

- We provide round the clock customer support

- Convenient online submission

- Thorough peer review

- Inclusion in PubMed and all major indexing services

- Maximum visibility for your research

Submit your manuscript at www.biomedcentral.com/submit 